\title{
ANÁLISIS DE LA VARIABILIDAD DEL CRECIMIENTO INFANTIL EN DIVERSOS AMBIENTES DE LA PROVINCIA DE BUENOS AIRES, ARGENTINA
}

\author{
ANALYSIS OF THE VARIABILITY OF CHILD GROWTH IN VARIOUS \\ ENVIRONMENTS OF THE BUENOS AIRES PROVINCE, ARGENTINA
}

\author{
María Antonia Luis ${ }^{1,2^{\star}}$, Fabián Aníbal Quintero1,3, María Fernanda Torres ${ }^{3,4}$, Luis Eduardo \\ Castro ${ }^{1}$, María Florencia Cesani³ ${ }^{3}$ María Laura Bergel ${ }^{3}$, Mariela Garraza ${ }^{3}$, Bárbara Navazo ${ }^{3}$ y \\ Evelia Edith Oyhenart ${ }^{1,3}$
}

\begin{abstract}
${ }^{1}$ Cátedra de Antropología Biológica IV, Facultad de Ciencias Naturales y Museo. Universidad Nacional de La Plata (UNLP). La Plata. Argentina

${ }^{2}$ Cátedra de Biología Humana. Facultad de Psicología. UNLP. Ensenada. Argentina

${ }^{3}$ Instituto de Genética Veterinaria (IGEVET), Centro Científico y Tecnológico La Plata (CCT), Consejo Nacional de Investigaciones Científicas y Técnicas (CONICET). Facultad de Ciencias Veterinarias. UNLP. La Plata. Argentina

${ }^{4}$ Facultad de Filosofía y Letras. Universidad de Buenos Aires (UBA). Ciudad Autónoma de Buenos Aires. Argentina
\end{abstract}

PALABRAS CLAVE crecimiento infantil; antropometría; heterogeneidad rural

\begin{abstract}
RESUMEN A fin de indagar la incidencia de los cambios acaecidos en los ámbitos urbano y rural, el objetivo del estudio fue caracterizar y comparar la expresión del crecimiento infanto-juvenil en los partidos bonaerenses de La Plata, Brandsen, Magdalena y Punta Indio. Se realizó un estudio antropométrico transversal en 7875 niños/as de 4,0-12,9 años de edad. Fueron relevados peso corporal, estaturas total y sentado, pliegues subcutáneos tricipital y subescapular, y se calculó el Índice de Masa Corporal. La información socio-ambiental de residencia fue obtenida mediante una encuesta autoadministrada. Para estimar diferencias entre partidos fueron calculadas frecuencias y comparadas mediante pruebas de Chi2 y Tukey. Los datos antropométricos
\end{abstract}

fueron analizados aplicando modelo lineal, considerando edad, sexo y localidad y sus interacciones. Los resultados indicaron que los niños de Punta Indio presentaron mayor tamaño corporal y ponderal junto con mayor reserva adiposa subcutánea, al tiempo que mayor porcentaje de padres y madres contaban con trabajo formal y niveles educativos más altos. Se concluye que Brandsen, Punta Indio y Magdalena, circundantes al área metropolitana de La Plata, presentan sensibles diferencias socio-ambientales, aunque compartan semejanzas en el paisaje y tradición agropecuaria. Esa heterogeneidad se vería reflejada en la variabilidad del crecimiento de la población infantil. Rev Arg Antrop Biol 20(1), 2018. doi:10.17139/raab.2018.0020.01.04

KEY WORDS child growth; anthropometry; rural heterogeneity

ABSTRACT The aim of this study was to characterize and compare changes in child and adolescent growth in the districts of La Plata, Brandsen, Magdalena, and Punta Indio, province of Buenos Aires, Argentina, and determine their impact in urban and rural areas. A cross-sectional, anthropometric study was performed on 7,875 children aged 4.012.9 years. Body weight, standing and sitting height, tricipital and subscapular subcutaneous skinfolds were measured, and body mass index was calculated. Socio-environmental information regarding residence was obtained through a selfadministered survey. Differences among districts were calculated with frequencies, and data were compared using Chi2 and Tukey Tests. Anthropometric data were analyzed using linear model considering the variables age, sex, and district and their interactions. The results indicated that children from Punta Indio had greater weight and body size, greater subcu-

El crecimiento humano y los patrones de desarrollo reconocen una base biológica en interacción sinérgica con factores sociales, económicos, políticos y ambientales propios del lugar de residencia. En este sentido, Bogin (2000) enfatizó en la necesidad de un enfoque biocul- taneous fat reserves, and a higher percentage of fathers and mothers had formal employment and a higher educational level. We can therefore conclude that Brandsen, Punta Indio, and Magdalena, all surrounding the metropolitan area of $\mathrm{La}$ Plata, present considerable socio-environmental differences, although they share similarities in their landscapes as well as in their agricultural traditions. Such heterogeneity might be reflected in growth changes in the child and adolescent population. Rev Arg Antrop Biol 20(1), 2018. doi:10.17139/ raab.2018.0020.01.04

*Correspondencia a: María Antonia Luis. Cátedra de Antropología Biológica IV, Facultad de Ciencias Naturales y Museo, Universidad Nacional de La Plata. Calle 64 Nº 31900 La Plata. Buenos Aires. Argentina. E-mail: maluis@fcnym.unlp.edu.ar

Financiamiento: Universidad Nacional de La Plata (Proyecto 11/N552), Agencia Nacional de Promoción Científica y Tecnológica (PICT 01541) y Consejo Nacional de Investigaciones Científicas y Técnicas (PIP 02197).

Recibido 20 Abril 2017; aceptado 12 Julio 2017

doi:10.17139/raab.2018.0020.01.04 
tural del crecimiento y desarrollo humano, a fin de abordar de un modo integral los diferentes aspectos que ese proceso involucra. La naturaleza compleja del enfoque permite considerar al cuerpo humano como lugar de expresión de posibles condiciones de desigualdad e inequidad (embodiment) que puede enfrentar una sociedad (Nguyen y Preschard, 2003).

Una de las formas más sencillas y reproducibles para describir el crecimiento humano es la antropometría. Las medidas más frecuentemente utilizadas son el peso, la talla o estatura, los pliegues subcutáneos y el perímetro braquial (WHO, 2006). El estudio y análisis de estas variables provee, no sólo, importante información acerca del bienestar biológico sino también de aspectos vinculados a la situación general de la sociedad y de su economía (Tanner, 1987). Así, por ejemplo, la estatura es una de las variables que mejor refleja el crecimiento y la salud de una población, pero además es considerada un potente indicador de la calidad de vida, incluso mejor que otros indicadores económicos como el Producto Bruto Interno per cápita (PBI) que, al verse afectado por la distribución del ingreso, aun cuando aumente no se refleja necesariamente en una mejora para la mayor parte de la población (Meisel y Vega, 2006). En consecuencia, el reconocimiento de la convergencia de factores socioeconómicos y biológicos, en el curso del proceso de crecimiento y desarrollo humano, ha promovido el surgimiento de abordajes interdisciplinarios y el nacimiento de la antropometría histórica como campo de investigación científica (Fogel et al., 1982).

En relación con lo expresado, numerosas investigaciones se han abocado al estudio de diferencias urbano-rurales en el crecimiento infantil y demostraron que, en general, los niños urbanos son menos propensos a presentar retardo en el crecimiento lineal y ponderal (Fox y Heaton, 2012; Paciorek et al., 2013; Zong y Li, 2014). Gran parte de estos estudios se sustentan en la idea de que el ambiente urbano ofrece a sus habitantes mayores posibilidades económicas y sociales que se traducen en mejores condiciones de vida para el desarrollo de una vida saludable. No obstante, la globalización ha cambiado las relaciones entre el campo y la ciudad con tal profundidad, que no sólo resulta necesario revisar dichos supuestos sino también analizar la situación actual en el marco de los cambios históricos particulares. En este contexto de referencia consideramos de interés analizar las condiciones que se revelan en algunos partidos de la provincia de Buenos Aires (Argentina).

La provincia de Buenos Aires forma parte de la región pampeana, junto con las provincias de Córdoba, Entre Ríos, Santa Fe y La Pampa. Desde un punto de vista económico, dicha provincia es una de las más importantes del territorio argentino, ya que sus características climáticas y edafológicas la transforman en una zona agrícola y ganadera por excelencia, con un aporte de, aproximadamente, el 36\% del PBI nacional (Ministerio del Interior, 2017). También lo es, demográficamente, ya que en ella vive el $38 \%$ de la población total del país y presenta un alto grado de urbanización. Está conformada por 135 partidos que, en términos de extensión geográfica, tamaño de la población, tipo y concentración de la actividad económica y situación social, evidencia importantes diferencias. En consecuencia, aun cuando es habitual el reconocimiento de la diversidad entre partidos, en la práctica, se traduce en la diferenciación entre partidos centrales y del interior, entre zonas agrícolas y ganaderas o entre regiones geográficas (Quesada Aramburú y Cadelli, 2012).

En su devenir histórico, especialmente entre los años 1960-2001, la región pampeana ha sufrido profundos cambios socioeconómicos que desestabilizaron su estructura agraria y posibilitaron la conformación de un nuevo modelo denominado "nueva ruralidad" basado en la innovación tecnológica, la apertura comercial al exterior, la transformación de la institucionalidad pública y los modos de regulación de la producción y distribución de la riqueza (Echeverri Perico y Ribero, 2002). Surgieron, así, nuevos usos del suelo, alejados del clásico interés agro-ganadero de la región, que dieron origen a emprendimientos turísticos, recreativos y de nueva residencia alejada de la ciudad. Del mismo modo, se produjeron cambios en las dinámicas urbanas y el ámbito rural surgió como un escenario de compensación de los traumas urbanos en el sentido de reproducir antiguas formas de vida y costumbres con el fin de mitigar la anomia de la vida en las ciudades (García Ramón et al., 1995). Así, las transformaciones económicas, políticas y sociales 
producidas derivaron en cambios en el trabajo rural acompañados por un consecuente proceso de despoblamiento en, prácticamente, todos los partidos de la provincia de Buenos Aires (Gorenstein, 2008).

A partir de lo expuesto surgió la necesidad de indagar la incidencia de los cambios acaecidos en los ámbitos urbano y rural de diferentes partidos bonaerenses colindantes entre sí. El objetivo del presente estudio fue caracterizar y comparar la expresión del crecimiento infantojuvenil en las poblaciones de La Plata, Brandsen, Magdalena y Punta Indio.

\section{MATERIAL Y MÉTODOS}

\section{Áreas de estudio}

En este trabajo se ha tenido en cuenta una clasificación de los partidos bonaerenses, basada en la técnica de aglomerados que los agrupa según tres categorías de indicadores, a saber: a) geográfico-demográfico, en el que se considera la densidad poblacional; b) social, en el que se estima la población con necesidades básicas insatisfechas y c) productivos, tales como actividad manufacturera, área sembrada, cría de ganado, magnitud del sector primario, entre otros. Desde esta perspectiva fueron identificados seis agrupamientos: industrial, industrial mixto, oleaginoso, ganadero, cerealero y turístico (Quesada Aramburú y Cadelli, 2012).

Se tomó como centro de referencia del estudio el partido de La Plata con la ciudad homónima, capital de la provincia de Buenos Aires. Dicho partido pertenece al aglomerado industrial mixto y se ubica en el noreste de la provincia de Buenos Aires, a $60 \mathrm{~km}$ de distancia de la Ciudad Autónoma de Buenos Aires (capital de la República Argentina), limita al noreste con los partidos de Ensenada y Berisso, al noroeste con los de Berazategui y Florencio Varela, al sudoeste y sur con San Vicente y Coronel Brandsen y al sudeste con Magdalena. Presenta una superficie de $926 \mathrm{~km}^{2}$, lo que representa un $0,3 \%$ del total de la provincia de Buenos Aires y cuenta con una población de 654.324 habitantes según el Instituto de Estadísticas y Censos (INDEC, 2010). En la actualidad, el partido incluye además del casco fundacional, conocido como "casco urbano", a 18 localida- des dependientes de la administración municipal central (Fig.1).

El partido de Brandsen, se incluye en el aglomerado industrial mixto, es decir, con un sector industrial basado en el aprovechamiento de la transformación de las materias primas extraídas por el sector primario de la economía, la lechería y la industria frigorífica. Dista $57,7 \mathrm{~km}$ de la ciudad de La Plata y $85 \mathrm{~km}$ de la Ciudad Autónoma de Buenos Aires. Limita con los partidos de La Plata, San Vicente, General Paz, Chascomús y Magdalena. Posee una superficie de $1130 \mathrm{~km}^{2}$, de los cuales un $90 \%$ están dedicados a la producción agrícola-ganadera y tambera. Cuenta con una población de 26.367 habitantes de los cuales el $85 \%$ reside en la ciudad cabecera del partido (ciudad de Brandsen), mientras que el resto se distribuye en áreas rurales y pequeños centros rurales de servicios (poblaciones con menos de 2000 habitantes) (INDEC, 2010) (Fig. 1).

El partido de Punta Indio pertenece al agrupamiento ganadero porque la principal actividad económica es la cría de ganado, aunque también registra alguna presencia de agricultura, soja, trigo y pasturas para la cría de bovinos. Dista $86 \mathrm{~km}$ de la ciudad de La Plata y $140 \mathrm{~km}$ de la ciudad de Buenos Aires. Limita al norte con el partido de Magdalena, al este con el Río de La Plata, al sur con la Bahía de Samborombón y al oeste con el partido de Chascomús. Tiene una superficie de $1627 \mathrm{~km}^{2}$, con una población de 9964 habitantes y una densidad poblacional de 6,1 habitantes $/ \mathrm{km}^{2}$ (INDEC, 2010) (Fig. 1).

El partido de Magdalena, al igual que el de Punta Indio, se ubica en el aglomerado ganadero. Posee $1863 \mathrm{~km}^{2}$ que representan el $0,58 \%$ de la superficie provincial. Ubicada en el noreste del territorio bonaerense, limita con el partido de La Plata al norte, con el Río de la Plata al este, con el partido de Chascomús al oeste y con el partido de Punta Indio al sur. Según últimos datos censales la población del partido asciende a 19.301 habitantes (INDEC, 2010). A excepción de la ciudad de Magdalena, cabecera del partido, las restantes localidades tienen una baja demografía poblacional, estimada en 10,4 habitantes $/ \mathrm{km}^{2}$ (INDEC, 2010). Este partido y el de Punta Indio se caracterizan a nivel provincial por tener una baja densidad poblacional (Quesada Aramburú y Cadelli, 2012) (Fig. 1). 


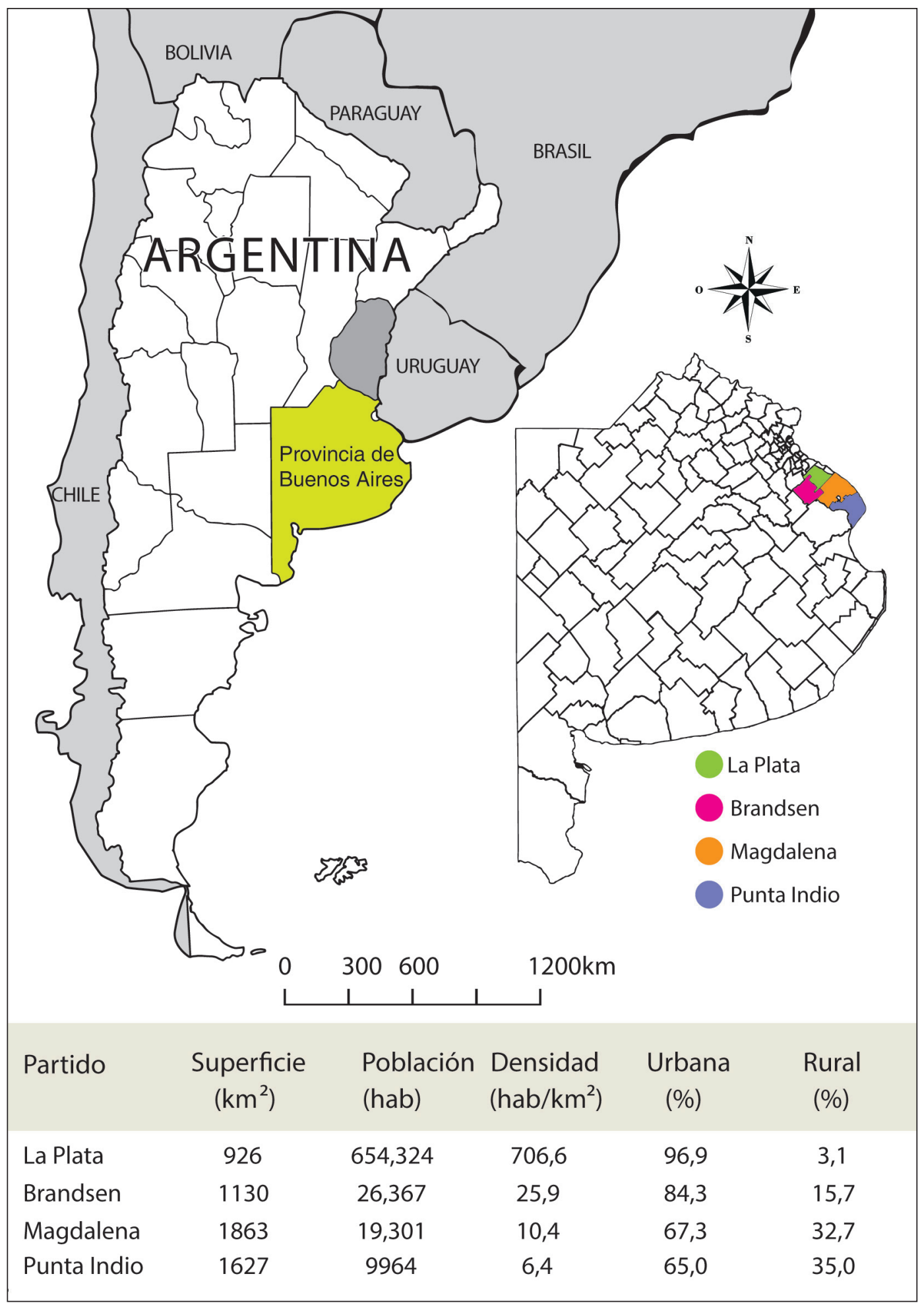

Fig.1. Ubicación geográfica y datos poblacionales de los partidos de La Plata, Brandsen, Magdalena y Punta Indio (Buenos Aires, Argentina). 


\section{Diseño del estudio y población}

Se realizó un estudio cuantitativo y transversal que incluyó escolares de 4 a 12 años de edad. Los establecimientos educativos en los que se efectuó el estudio fueron seleccionados de manera no aleatoria a partir de un muestreo por conveniencia. Se excluyeron los niños con antecedentes patológicos, sin autorización escrita de los padres/tutores y aquellos que manifestaron su negativa a participar. La muestra quedó conformada por 7875 niños y niñas (Tabla 1). El estudio se llevó a cabo durante los ciclos lectivos comprendidos en el período 2008-2012 e incluyó los siguientes ejes de análisis:

\section{Estudio socio-ambiental}

Se realizó una encuesta estructurada y autoadministrada a los padres o tutores. Se relevaron datos del ambiente intradomiciliario (características constructivas de la vivienda y hacinamiento crítico) y peridomiciliario (disponibilidad de servicios: pavimento, agua corriente, energía eléctrica, recolección de residuos, cloacas, gas natural por red). Para complementar la información sobre el nivel socioeconómico familiar, se consideraron los siguientes aspectos: régimen de tenencia de la vivienda, nivel de instrucción y empleo de los padres, cobertura médica de salud, prácticas de cultivo en huertas fa- miliares, cría de animales y acceso a bienes materiales y de consumo, planes sociales y/o programas alimentarios (Oyhenart et al., 2008) (Tabla 2).

\section{Estudio antropométrico}

Las mediciones fueron realizadas por antropometristas entrenados, de acuerdo a protocolos estandarizados (Lohman et al., 1988). El peso corporal (P) se midió en kilogramos con una balanza digital portátil (100g de precisión) que se calibró al inicio de cada sesión. En todos los casos los niños vistieron ropa liviana cuyo peso se descontó del peso total. La talla (T) se midió en centímetros con un antropómetro vertical ( $1 \mathrm{~mm}$ de precisión) con el niño descalzo y la cabeza orientada en el plano de Frankfort. La estatura sentado (ES) se midió en centímetros con antropómetro vertical (1 $\mathrm{mm}$ de precisión) y correspondió a la distancia entre el Vértex al plano en el que el niño estaba sentado. El perímetro braquial (PB) se midió en centímetros con cinta métrica inextensible ( $1 \mathrm{~mm}$ de precisión) y los pliegues subcutáneos tricipital (PT) y subescapular (PS) en milímetros con calibre Lange de presión constante (1mm de precisión). La edad de cada niño se obtuvo del documento nacional de identidad y en función de su fecha de nacimiento se calculó la edad exacta (decimal).

TABLA 1. Distribución de la muestra, en varones y mujeres, por partido de procedencia y edad

\begin{tabular}{|c|c|c|c|c|c|c|c|c|c|c|c|c|}
\hline \multirow{2}{*}{$\begin{array}{c}\text { Edad } \\
\text { (años) }\end{array}$} & \multicolumn{3}{|c|}{ La Plata } & \multicolumn{3}{|c|}{ Brandsen } & \multicolumn{3}{|c|}{ Magdalena } & \multicolumn{3}{|c|}{ Punta Indio } \\
\hline & Varón & Mujer & Total & Varón & Mujer & Total & Varón & Mujer & Total & Varón & Mujer & Total \\
\hline $4,04,9$ & 258 & 234 & 492 & 110 & 114 & 224 & 27 & 26 & 53 & 29 & 37 & 66 \\
\hline $5,05,9$ & 313 & 270 & 583 & 121 & 117 & 238 & 30 & 22 & 52 & 50 & 53 & 103 \\
\hline $6,06,9$ & 323 & 317 & 640 & 65 & 62 & 127 & 23 & 18 & 41 & 37 & 35 & 72 \\
\hline $7,07,9$ & 307 & 291 & 598 & 60 & 55 & 115 & 20 & 24 & 44 & 45 & 31 & 76 \\
\hline $8,08,9$ & 336 & 349 & 685 & 37 & 52 & 89 & 20 & 25 & 45 & 28 & 51 & 79 \\
\hline $9,09,9$ & 379 & 347 & 726 & 47 & 69 & 116 & 15 & 13 & 28 & 53 & 39 & 92 \\
\hline $10,0 \quad 10,9$ & 321 & 368 & 689 & 50 & 75 & 125 & 11 & 20 & 31 & 35 & 43 & 78 \\
\hline $11,011,9$ & 307 & 324 & 631 & 55 & 51 & 106 & 23 & 24 & 47 & 37 & 31 & 68 \\
\hline $12,012,9$ & 245 & 277 & 522 & 47 & 56 & 103 & 17 & 19 & 36 & 20 & 35 & 55 \\
\hline Total & 2789 & 2777 & 5566 & 592 & 651 & 1243 & 186 & 191 & 377 & 334 & 355 & 689 \\
\hline
\end{tabular}


M.A. LUIS ET AL./REV ARG ANTROP BIOL 20(1), 2018. doi:10.17139/raab.2018.0020.01.04

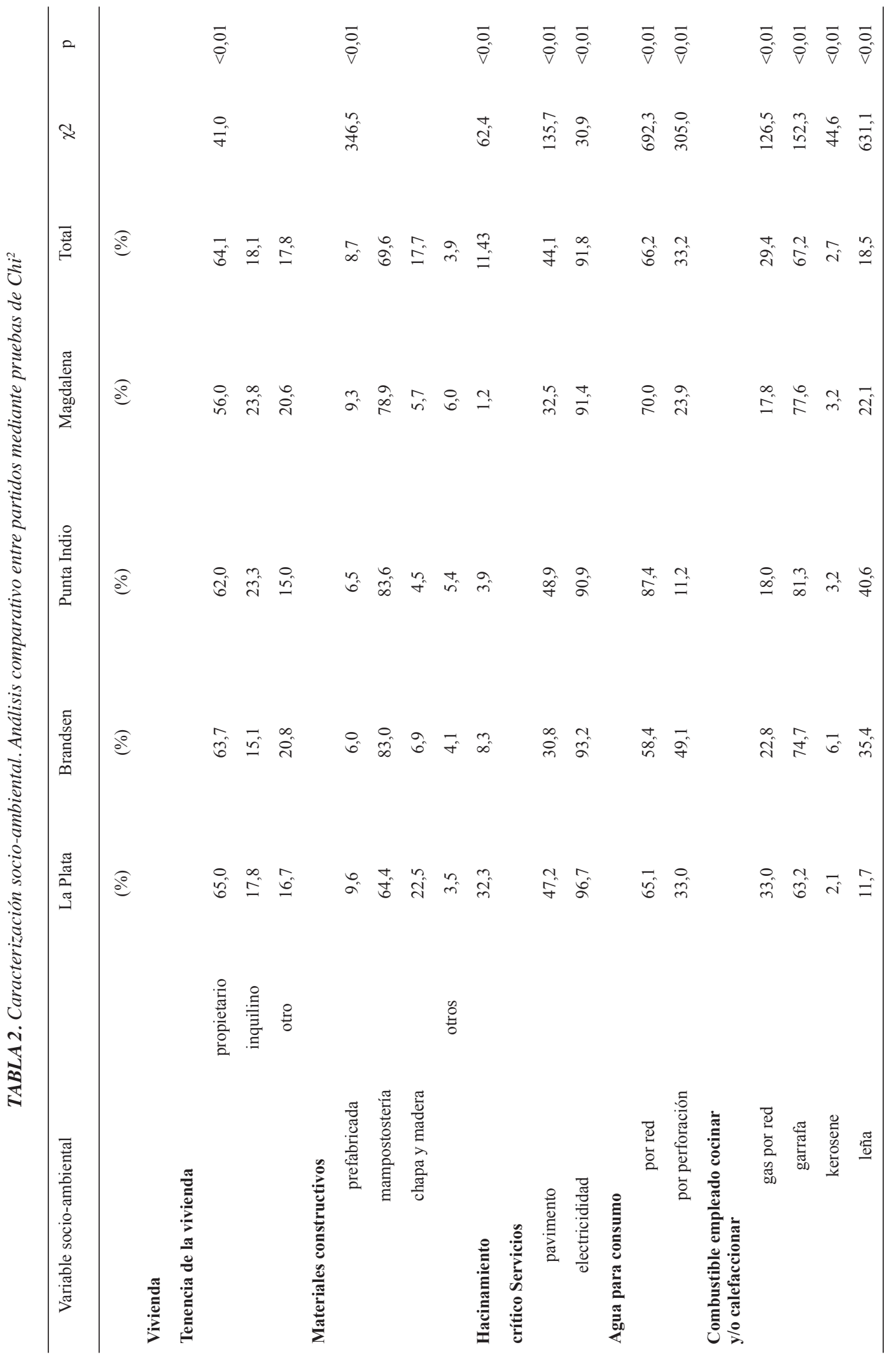


CRECIMIENTO INFANTIL EN LA PROVINCIA DE BUENOS AIRES

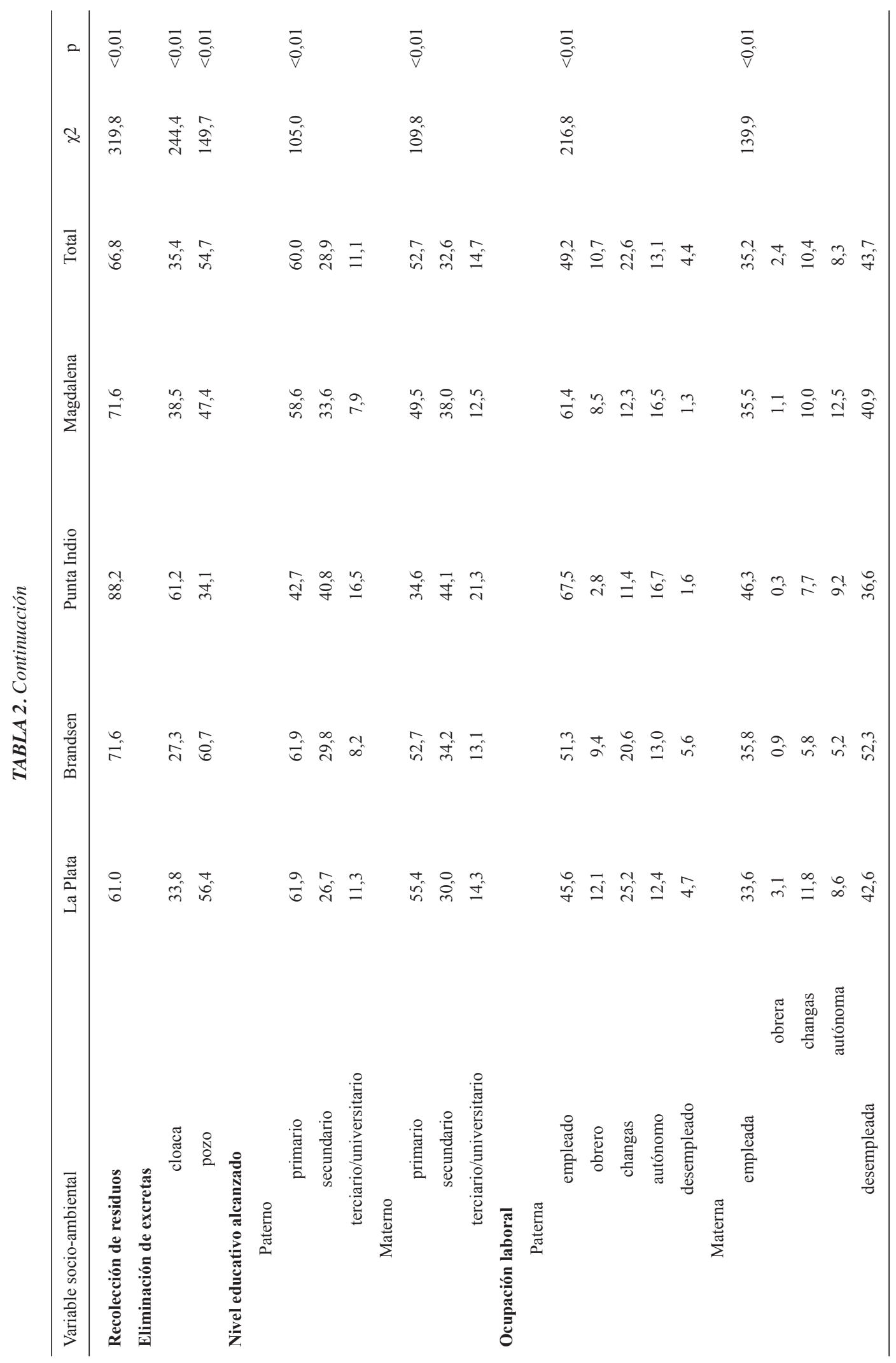




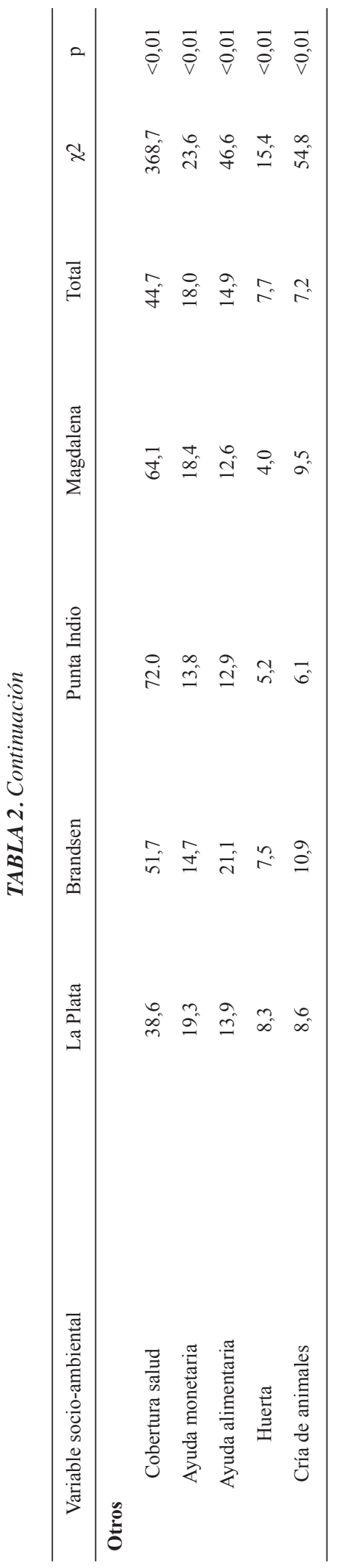

\section{Procesamiento estadístico}

Para estimar diferencias de las características socio-ambientales de residencia de las familias fueron calculadas frecuencias que se compararon mediante pruebas de Chi cuadrado.

Los datos antropométricos se analizaron mediante modelos de regresión y se estimaron los valores para las variables teniendo en cuenta los factores sexo, edad y partido de residencia. Para la comparación entre partidos se tomó a La Plata como referencia por ser la capital de la provincia y sede administrativa de Buenos Aires. Posteriormente, se realizaron comparaciones entre los restantes partidos aplicando pruebas de Tukey. A partir de los modelos de regresión se calcularon y graficaron las diferencias entre partidos, para cada variable. Para todas las pruebas se utilizó un nivel de significación menor a 0,05.

\section{Consideraciones éticas}

Todos los datos personales fueron resguardados en orden a lo prescripto en las normativas y reglamentaciones bioéticas vigentes, observando el estricto cumplimiento de la Ley Nacional Argentina $\mathrm{N}^{\mathrm{o}}$ 25.326/00 y su reglamentación No $^{\mathrm{1}} 1558 / 01$. El presente trabajo surge de un proyecto marco que fue evaluado y aprobado por el Comité de Bioética de la Escuela Latinoamericana de Bioética (CE.LA.BE).

\section{RESULTADOS}

\section{Estudio socio-ambiental}

El análisis comparativo de las condiciones socio-ambientales de los partidos indicó diferencias significativas para todas las variables analizadas. Las familias de Punta Indio presentaron, en general, las mejores condiciones de saneamiento, infraestructura y nivel socioeconómico y las de Brandsen, las menos favorables (Tabla 2).

Con respecto al régimen de tenencia de la vivienda, La Plata presentó mayor porcentaje de propietarios, sin embargo, registró la menor calidad constructiva de las mismas dada por el mayor porcentaje de construcción con chapa y madera. El hacinamiento crítico, condición representada por la presencia de 3 ó más habitantes por cuarto, también fue muy superior en 
La Plata. En contraposición, en Punta Indio las viviendas fueron de mejor calidad constructiva y a excepción de la provisión de gas por red, tuvieron las mayores prestaciones de servicio: pavimento, agua por red, cloacas y recolección de residuos (Tabla 2).

En cuanto al nivel educativo alcanzado, tanto por los padres como por las madres, los mayores porcentajes para nivel secundario y terciario-universitario correspondieron a los residentes en Punta Indio. Los porcentajes más bajos para nivel secundario se registraron en La Plata y para nivel terciario-universitario en Magdalena. Por otra parte, el mayor porcentaje de padres y madres con trabajo formal (empleado, obrero y autónomo) se observó en Punta Indio y Magdalena. Comparativamente, el porcentaje de trabajadores ocasionales y desempleados fue mayor en La Plata y Brandsen (Tabla 2).

Con relación a la cobertura de salud, Punta Indio presentó el mayor porcentaje de familias con acceso a dicho servicio mientras que La Plata, el menor. La ayuda monetaria fue mayor en La Plata y menor en Punta Indio, en tanto que la ayuda alimentaria fue mayor en Brandsen y semejante en los otros partidos. El menor porcentaje de familias con huertas se presentó en Magdalena y el mayor en La Plata. La cría de animales, para consumo familiar, fue mayor en Brandsen y menor en Punta Indio (Tabla 2).

\section{Estudio antropométrico}

Los resultados obtenidos de la aplicación del modelo lineal indicaron que las variables peso corporal, talla, estatura sentado, perímetro braquial y pliegues subcutáneos tricipital y subescapular aumentaron con la edad (Tabla 3).

La comparación entre sexos indicó diferencias significativas con valor negativo para peso corporal, talla y estatura sentado y positivo para pliegues tricipital y subescapular. El perímetro braquial no se diferenció (Tabla 3).

La comparación entre los niños y niñas, tomados en su conjunto, residentes en los diferentes partidos indicó que: los de Punta Indio con relación a los de La Plata presentaron diferencias significativas con valores positivos para peso corporal, talla, estatura sentado, perímetro braquial y pliegue tricipital. Los de Brandsen en cambio, se diferenciaron de los de La Plata en

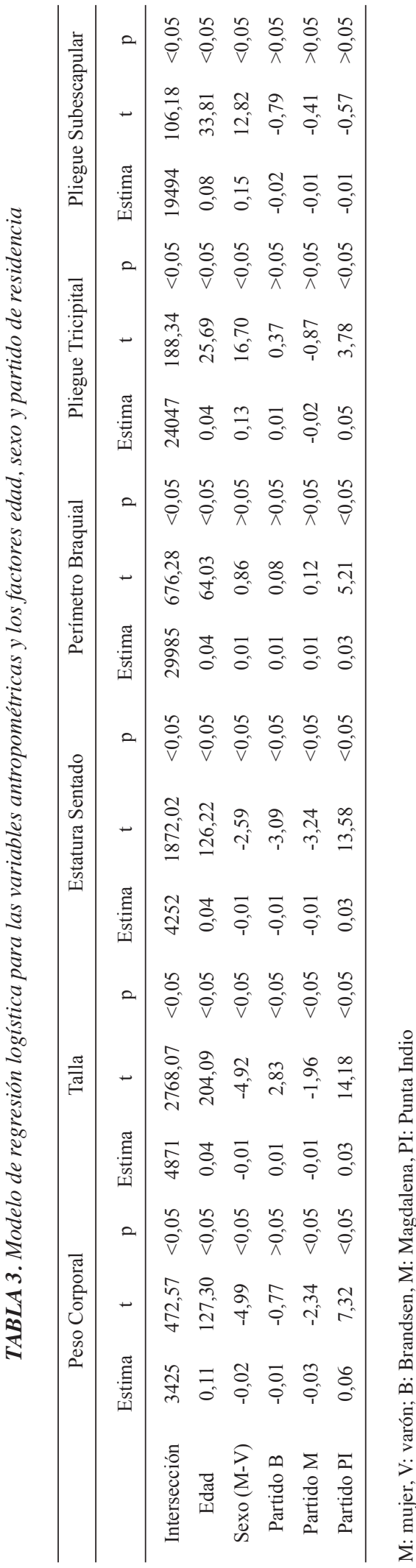




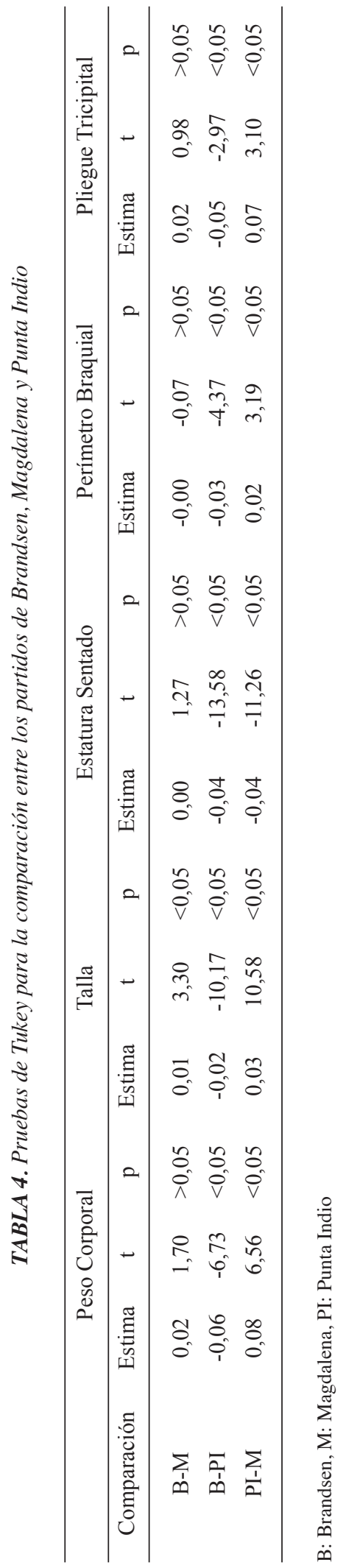

la talla (valores positivos) y la estatura sentado (valores negativos) y los de Magdalena en peso corporal, talla y estatura sentado (valores negativos). En razón de que el pliegue subcutáneo subescapular mostró diferencias no significativas no fue incluido en la Figura 2 (Tabla 3; Fig. 2).

Por último, las comparaciones entre los niños y niñas de Brandsen (en su conjunto) respecto a los de Punta Indio indicaron diferencias significativas y valores negativos para peso corporal, talla, estatura sentado, perímetro braquial y pliegue tricipital y respecto de los de Magdalena sólo para talla con valor positivo (Tabla 4; Fig. 2). La comparación entre los niños de Punta Indio y Magdalena indicó diferencias significativas con valores positivos para peso corporal, talla, perímetro braquial y pliegue tricipital y negativo para estatura sentado (Tabla 4; Fig. 2).

\section{DISCUSIÓN}

La caracterización socio-ambiental y del crecimiento infanto-juvenil de las poblaciones estudiadas permitió establecer diferencias entre las mismas. Considerando las condiciones ambientales de la población de La Plata se observa un franco deterioro, en aspectos vinculados a la calidad de vida, puesto que se posicionó desventajosamente respecto a la presencia de servicios, instrucción y ocupación de los padres, debido, tal vez, a la creciente urbanización de los suburbios o periurbano. Por otra parte, los partidos circundantes al área metropolitana también se diferenciaron entre sí, aun cuando constituyen espacios colindantes relativamente similares en cuanto a paisaje y tradición agropecuaria. Dichas diferencias inducen a aceptar una resignificación de la ruralidad frente a las intencionalidades de homogeneización pretendidas por la globalización (Ruiz Rivera y Delgado Campos, 2008).

Al menos para Argentina, la realidad socioespacial evidencia que lo rural y lo agrario ya no significan lo mismo. A partir de 1990, la región pampeana experimentó un proceso de intensificación y expansión de la agricultura a partir de la incorporación masiva de nuevas tecnologías, el cultivo de semillas transgénicas y la difusión de la siembra directa (Navarrete y Gallopin, 2007). Este proceso denominado "agriculturización" ocasionó un fuerte impacto económico y social en las comunidades rurales de la región 

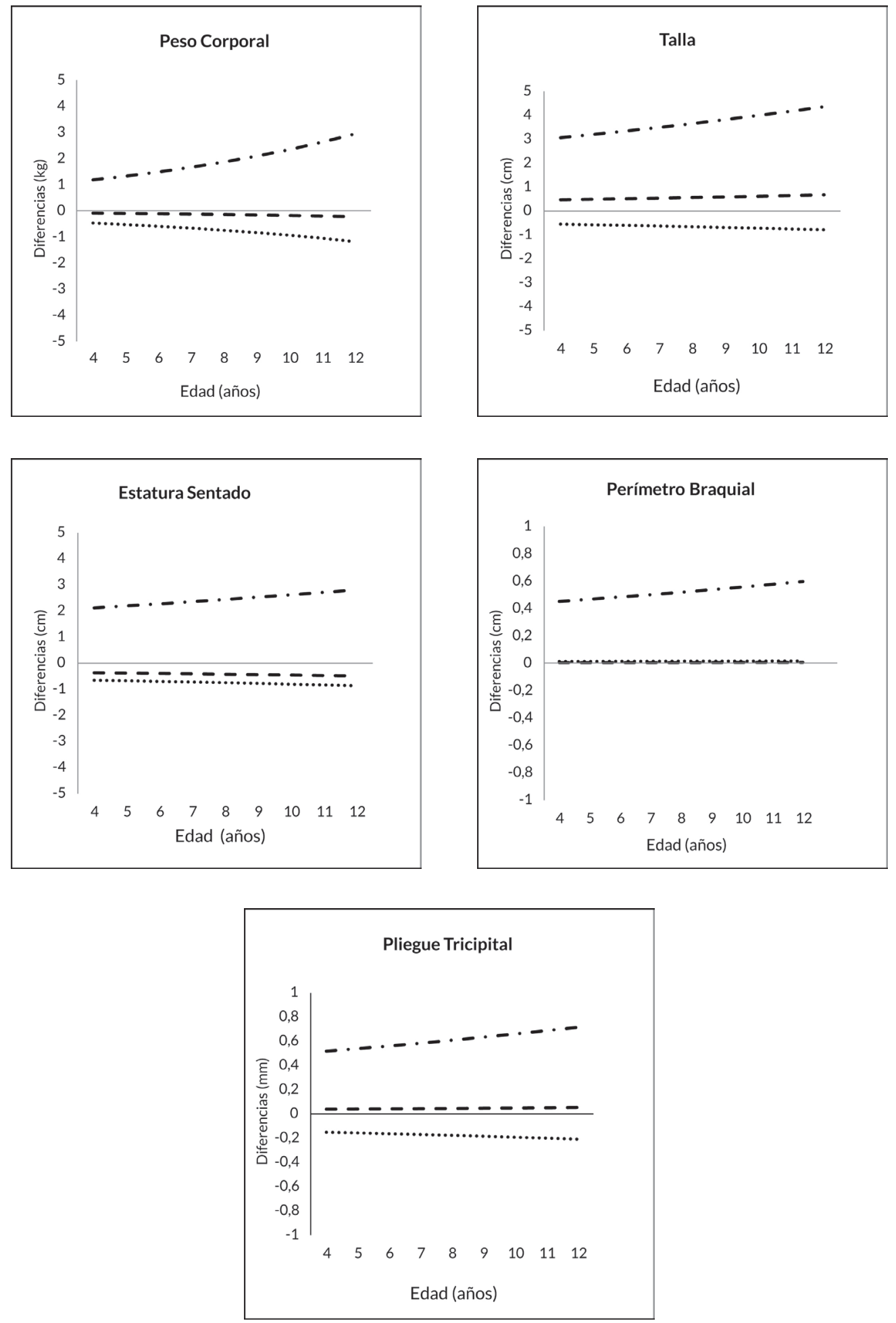

Fig. 2. Cuantificación de las diferencias por edad entre niños de ambos sexos en su conjunto de los partidos bonaerenses de Brandsen (- - - ), Magdalena (•••) y Punta Indio ( - • - ) respecto de La Plata. 
que impulsó el aumento de la productividad agropecuaria pero también de una agricultura sin agricultores, con el marcado despoblamiento en las áreas rurales, en muchos casos, con riesgo de desaparición (Rabinovitch y Torres, 2004). Este nuevo modelo generó una alta concentración de la riqueza en pocos productores y una profunda crisis en los pequeños productores anclados sobre la base del trabajo familiar en el campo. Como consecuencia, se generó una verdadera fragmentación social de la comunidad rural de la región pampeana (Gras, 2012).

Los partidos rurales que rodean al área metropolitana de La Plata registraron, no sólo, grandes transformaciones en las propias prácticas agropecuarias sino también reestructuraciones en los mercados de bienes, servicios y trabajo. Contribuyeron a ello la difusión de lo urbano, la movilidad poblacional y los nuevos hábitos de consumo y estilos de vida, dando lugar a una nueva dinámica en su estructura. Así, la creciente diversificación de usos y actores puede explicar el proceso de heterogeneización que atraviesan los ámbitos rurales, situación que se reflejaría en el crecimiento de los niños que allí residen.

Las condiciones socio-ambientales de residencia de las familias de Punta Indio, respecto a la de los otros partidos, revelaron aspectos más favorables en el saneamiento, la infraestructura y el nivel socioeconómico. En cambio, las menos favorables se presentaron en La Plata y Brandsen, en tanto que Magdalena reflejó una situación intermedia. Punta Indio, al igual que la mayoría de los partidos de Buenos Aires, se enfrentó a la amenaza de desertificación social por el proceso de despoblamiento de las zonas rurales. Ello así porque su economía, básicamente agropecuaria, con énfasis en la ganadería, con un sector industrial y de servicios poco desarrollados contribuyó al proceso de éxodo particularmente de los jóvenes y de las familias de productores que migraron a los centros urbanos. En este sentido, con el objetivo de minimizar dicho proceso se iniciaron acciones basadas en los alcances del programa LEADER (Liaisons entre activités de Developement de L'Economie Rural) ejecutado en varios países de la Unión Europea con problemáticas de despoblamiento rural (De los Ríos-
Carmenado, 2002). Dicha iniciativa incluyó la participación de la comunidad a través de distintas actividades que condujo a la formación de un Grupo de Acción Local, integrado por representantes de la comunidad y autoridades municipales, responsable de la fijación de prioridades y diseño de proyectos para satisfacerlas. En este sentido, se iniciaron acciones vinculadas al sector turismo, favorecidas por las condiciones del lugar. Así, Punta Indio se encuentra dentro del Parque Costero del Sur declarado, en 1984, por la UNESCO (United Nations Educational, Scientific and Cultural Organization) reserva mundial de la biósfera natural y cultural. La potencialidad de este sector turístico permitió el desarrollo de numerosos proyectos como alternativa de capacitación y ocupación de los jóvenes, entre otros emprendimientos (Stratta Fernández et al., 2013).

Los resultados obtenidos en este trabajo son coincidentes con los hallados en estudios realizados por el Departamento de Educación para la Salud y Bienestar, en la población estadounidense, en tanto demostraron que son las condiciones socio-económicas las que influyen en las diferencias en el crecimiento de los niños rurales con relación a los de las ciudades, ya que al no considerar en la muestra a los niños de familias de bajos ingresos las diferencias encontradas desaparecían (Hamill et al., 1979). En el mismo sentido, otras investigaciones realizadas en Estados Unidos, Holanda y Australia han demostrado que las diferencias observadas devienen insignificantes o han desaparecido debido al mejoramiento de las condiciones de vida en las zonas rurales (Eveleth, 1986). Se ha observado además que las diferencias registradas en el crecimiento de niños rurales y urbanos tienden a aumentar en países económicamente menos desarrollados como Costa Rica (Villarejos et al., 1971), México (Tena-Flores y Frisancho, 1997; Malina et al., 2008), Venezuela (López de Blanco y Landaeta-Jiménez, 2003), Sud-África (Cameron et al., 1992) y Nigeria (Ayoola et al., 2009). Resulta entonces que el crecimiento más favorable de los niños de Punta Indio -reflejado en un mayor tamaño corporal y ponderal, acompañado por una mayor reserva adiposa subcutánea- encontraría explicación 
en las condiciones socio-ambientales más propicias en las que dicho proceso se desarrolló. En este sentido en Punta Indio, son particularmente considerables las mejoras alcanzadas en el tipo de trabajo y en el nivel educativo de los padres, que difieren sistemáticamente de los otros contextos de residencia.

La expresión del crecimiento a largo plazo dada por la talla, exhibió claramente este bienestar en los niños de Punta Indio. Sin embargo, la presencia de mayor adiposidad dio cuenta del riesgo potencial para la salud y del incremento de exceso de peso, tal como fuera comunicado por Bergel Sanchís et al. (2016). Tanto La Plata como Brandsen, partidos con mayor densidad de población, presentan un periurbano donde las condiciones socioeconómicas familiares y de saneamiento ambiental son deficientes y posicionan desventajosamente a los niños que allí crecen (Cesani et al., 2007; Zonta et al., 2007; Bergel et al., 2011; Oyhenart et al., 2013). Por último, en el partido de Magdalena con menor densidad demográfica y condiciones socio-ambientales intermedias, el crecimiento infantil se asemejó más al observado en La Plata y Brandsen, diferenciándose claramente de la población vecina de Punta Indio.

\section{CONCLUSIONES}

Aunque constituyen espacios relativamente similares en cuanto a paisaje y tradición agropecuaria, los partidos bonaerenses de Brandsen, Punta Indio y Magdalena, colindantes al área metropolitana de la ciudad de La Plata, registran sensibles diferencias socio-ambientales. Esa heterogeneidad se ve reflejada en la variabilidad del crecimiento de la población infantil.

\section{AGRADECIMIENTOS}

A los niños y sus padres que participaron desinteresadamente en el trabajo de investigación. A las autoridades escolares y maestros que facilitaron nuestro trabajo de campo en los establecimientos educativos seleccionados para el estudio. A las instituciones que brindaron su apoyo financiero. A Lucía Featherston por el diseño de las figuras y a Adriana Di Maggio por la revisión idiomática del inglés.

\section{LITERATURA CITADA}

Ayoola O, Ebersole K, Omotade OO, Tayo BO, Brieger WR, Salami K, Dugas LR, Cooper RS, Luke A. 2009. Relative height and weight among children and adolescents of rural southwestern Nigeria. Ann Hum Biol. 36 (4):388399. doi:10.1080/03014460902835606.

Bergel ML, Garraza M, Luis MA, Torres MF, Castro LE, Quintero FA, Luna ME, Cesani MF, Oyhenart EE. 2011 Malnutrición y factores socio-económicos familiares en escolares del partido de La Plata (Buenos Aires, Argentina). En: Anthopos 2011. La Antropología ante los desafíos del Siglo XXI. Convención Internacional de Antropología. La Habana.

Bergel Sanchis ML, Quintero FA, Navazo B, Cesani MF, Garraza M, Torres MF, Luna ME, Luis MA, Castro, Oyhenart EE. 2016 Caracterización del estado nutricional en relación con factores socio-ambientales de la población escolar del partido de Punta Indio (Provincia de Buenos Aires, Argentina). Rev Arg Antrop Biol 18 (2): 1-13. doi: 10.17139/raab.2016.0018.02.09

Bogin B. 2000. Evolution of human life cycle. En: Stinson S, Bogin B, Huss-Ashmore R, O'Rourke D, editores. En: Human biology: an evolutionary and biocultural perspective. Nueva York Wiley-Liss, Inc.

Cameron NJ, Kgamphe S, Leschner KF, Farrant PJ. 1992 Urban-rural differences in the growth of South Africa black children. Ann Hum Biol 19 (1):23-33.

Cesani MF, Zonta ML, Castro LE, Torres MF, Forte LM, Orden AB, Quintero FA, Luis MA, Sicre ML, Navone GT, Gamboa MI, Oyhenart EE. 2007. Estado nutricional y parasitosis intestinales en niños residentes en zonas urbana, periurbana y rural del partido de Brandsen (Buenos Aires, Argentina). Rev Arg Antrop Biol 9 (2):105-121.

De los Ríos-Carmenado I. 2002 Innovación para el desarrollo rural: La iniciativa Leader como laboratorio de aprendizaje. Madrid: Editorial Agrícola.

Echeverri Perico R, Ribero MP. 2002. Nueva ruralidad. Visión del territorio en América Latina y el Caribe. Salvador de Bahía: Instituto Interamericano de Cooperación para la Agricultura.

Eveleth PB. 1986. Population differences in growth: environmental and genetic factors. En Human growth: a comprehensive treatise. En: Falkner F, Tanner JM, editores. New York: Plenum Press.

Fogel RW, Engerman SL, Trusell J.1982. Exploring the uses of data on heigth: the analysis of long-term trends in nutrition, labor welfare and labor productivity. Social Science History 6 (4):401-421. doi:https://doi. org/10.1017/S0145553200023130

Fox K, Heaton TB. 2012. Child nutritional status by rural/urban residence: a cross-national analysis. J Rural Health 28 (4):380-391. doi:10.1111/j.1748-0361.2012.00408.x

García Ramón M, Tulla A, Valdovinos N. 1995. Geografía rural. Madrid: Editorial Síntesis. Colección Espacios y Sociedades.

Gorenstein S. 2008. Agricultura familiar pampeana: tramas, territorios y políticas. Rev Interdiscipl Est Agr 29 (2):1-21.

Gras C. 2012. Cambio agrario y nueva ruralidad: caleidoscopio de la expansión sojera en la región pampeana. Trabajo y Sociedad 18 (15): 7-24.

Hamill P, Drizd T, Johnson C, Reed R, Roche A, Moore W. 1979. Physical growth: National Center for Health Statistics percentiles. Am J Clin Nutr 32 (3):607-629.

INDEC. Instituto Nacional de Estadística y Censos. 2010. Censo Nacional de población, hogares y vivienda. Mi- 
nisterio de Economía de la Nación. Disponible en: http/ www.indec.gov.ar (28 marzo 2017).

Lohman T, Roche A, Martorell R. 1988. Anthropometric standardization reference manual. Illinois: Human Kinetics Books,

López de Blanco M, Landaeta-Jiménez M. 2003. La antropometría en el estudio del crecimiento y desarrollo físico. Experiencia venezolana. Rev Esp Nutr Comunitaria 9 (3):128-136.

Malina RT, Peña Reyes ME, Little BB. 2008. Secular change in the growth status of urban and rural school children aged 6-13 years in Oaxaca, southern Mexico. Ann Hum Biol 35 (5): 475-489.

Meisel RA, Vega MA. 2006. Los orígenes de la antropometría histórica y su estado actual. Cuadernos de Historia Económica y Empresarial (18):1-70.

Ministerio del Interior de la Nación. 2017. Disponible en: http//www.mininterior.gov.ar/municipios/gestion/regiones_archivos/Pampeana.pdf (28 marzo 2017).

Navarrete DM, Gallopin G. 2007. Integración de políticas, sostenibilidad y agriculturización en la Pampa Argentina y áreas extrapampeanas. Comisión Económica para América Latina y el Caribe (CEPAL). Serie: Seminarios y Conferencias, 50. Santiago de Chile.

Nguyen VK, Preschard K. 2003. Anthropology, inequality and disease: a review. Annu Rev Anthropol 32: 447474. doi:10.1 146/annurev.anthro.32.061002.093412

Oyhenart EE, Castro LE, Forte LM, Sicre ML, Quintero FA, Luis MA, Torres MF, Luna ME, Cesani MF, Orden AB. 2008. Socio-environmental conditions and nutritional status in urban and rural schoolchildren. Am J Hum Biol 20 (4):399-405. doi:10.1002/ajhb.20738

Oyhenart EE, Garraza M, Bergel ML, Torres MF, Castro LE, Luis MA, Forte LM, Gamboa MI, Zonta ML, Cesani MF, Quintero FA, Luna ME, Navone GT. 2013. Caracterización del estado nutricional, enteroparasitosis y condiciones socio-ambientales de la población infanto-juvenil del partido de La Plata. Rev Arg Antrop Biol 15 (1):47-60. doi:10.17139/ raab.2013.0015

Paciorek CJ, Stevens GA, Finucane MM, Ezzati M. 2013. Children's height and weight in rural and urban populations in low-income and middle-income countries: a systematic analysis of population-representative data. Lancet Glob Health 1:300-309. doi:10.1016/S2214109X(13)70109-8
Quesada Aramburú J, Cadelli E. 2012. Hacia una clasificación de los municipios bonaerenses. Documento de Trabajo DPEPE $N^{\circ}$ 04/2012. Disponible en: http://www.ec.gba.gov.ar/areas/estudios_proyecciones/documentos/DT\%204\%20Hacia\%20una \%20 clasificaci $\%$ C3\%B3n\%20de $\% 201$ os $\% 20$ municipios\%20bonaerenses.pdf (29 marzo 2017).

Rabinovitch J, Torres F. 2004. Caracterización de los síndromes de sostenibilidad del desarrollo. El caso de Argentina. Comisión Económica para América Latina y el Caribe (CEPAL). Serie: Seminarios y Conferencias 38, Santiago de Chile.

Ruiz Rivera N, Delgado Campos J. 2008. Territorio y nuevas ruralidades: un recorrido teórico sobre las transformaciones de la relación campo-ciudad. EURE 34 (102):77-95. doi:10.4067/S0250-71612008000200005

Stratta Fernández R, Gómez Gajardo F, Rodríguez Sáez P. 2013. Rural depopulation in the Pampean region of Argentina: intervention model. Cuad Desarro Rural, 10 (70):201-218.

Tanner JM. 1987. Growth as a mirror of the condition of society: secular trends and class distinctions. Acta Paediatr Jpn 29 (1):96-103.

Tena-Flores JA, Frinsacho R. 1997. Crecimiento antropométrico de la población escolar en las zonas rurales y suburbanas de Durango, México. Arch Latinoam Nutr 47 (2):105-109.

Villarejos VM, Osborne JA, Payne FP, Arguedas VJA. 1971. Heights and weights of children in urban and rural Costa Rica. J Trop Pediatr Environ Child Health 17 (1):31-43.

WHO. World Health Organization. 2006. Multicentre growth reference study group. WHO Child growth standards: Length/height-for-age, weight-for-age, weightfor-length, weight-for-height and body mass index-forage: methods and development. Ginebra: World Health Organization.

Zong X, Li H. 2014. Physical growth of children and adolescents in China over the past 35 years. Bull World Health Organ 92:555-564. doi:10.2471/BLT.13.126243

Zonta M.L, Navone GT, Oyhenart EE. 2007. Parasitosis intestinales en niños de edad preescolar y escolar: situación actual en poblaciones urbanas, periurbanas y rurales en Brandsen, Buenos Aires, Argentina. Parasitol Latinoam. 62:54-60. doi:10.4067/S071777122007000100009 